\title{
Penalty Corners in Field Hockey: A guide to success
}

Peter Laird* \& Polly Sutherland**

*4 Seton Place, Edinburgh, EH9 2JT

**39/5 West Bryson Road, Edinburgh, EH11 1BQ.

\begin{abstract}
The purpose of this study was to devise a suitable notational analysis system (video analysis combined with hand notation) to help coaches and players improve their performance during penalty corners in field hockey. The design of the system sought to highlight successful tactics, while eliminating the unsuccessful ones. Two hundred and fifty penalty corners from the 1998 field hockey World Cup in Holland were analysed using video analysis and a hand notation system. Results showed that most successful goals were scored from straight shots that were either flicked or undercut. The average number of touches which resulted in a straight shot/outright goal was found to be three. Unsuccessful goals occurred most often when the ball was saved by either the goalie or a defender or when the attacker lost possession. Most shots that were on target, resulted in a save. These shots were usually hit without the ball leaving the ground. This information could be used by coaches and players to concentrate a portion of training time to perfecting these successful tactics and discouraging the unsuccessful ones. The ability to score from penalty corners can be the determining factor in winning a match
\end{abstract}

\section{Introduction}

"The Netherlands, the masters of penalty corner conversion, scored 5 goals from a total of 6 penalty corners the y manufactured in the semi-final and finals against Germany and Spain. A rate of $83 \%$....Amazing. It was educating to see how the Dutch coach, Roeland Oltmans played his cards in using his options. His tricks and ugly surprises left the opponents confused and overpowered" (Jagday, 1998).

Penalty corners are extremely important and can contribute significantly to the outcome of matches. For this reason it is in the interest of coaches and players that they devise effective strategies to make the most of these potential scoring opportunities. So important were penalty corners considered to be as a goal scoring device, that during the 1998 field hockey World Cup specialist penalty corner players were kept on the bench 
and brought on to substitute the other players when a penalty corner was awarded. This tactic has also been used for defensive short corners to reduce the oppositions chance of scoring. This serves to demonstrate how seriously coaches and players regard penalty corners as a successful tool for goal scoring.

Since then, the International Hockey Federation (FIH) has taken steps to disallow this practice so teams can no longer make player substitutions immediately after the award of a short corner (except to replace an injured player). The refore it is important that effective strategies and techniques are devised for penalty corners, which would allow the team on the field to perform the full range of hockey skills as effectively as specialist substitutes.

If basic statistical data are required by the coach and players, computer and videotape are of enormous value provided that the ground rules have been clearly identified and working criteria well established in the preparation stages (MacHeath, 1987). From such state of the art data the coach and the players have clear-cut evidence of success and failure against those opponents in the prevailing conditions.

One of the areas of greater use of such statistical data is at penalty corner play (MacHeath, 1987). At a set play a great deal of valuable data can be observed and utilised. Tactics in both attack and defence can be assessed, players' roles determined, strengths and weaknesses identified, and skills highlighted not only for the coach's own team but also for future opponents. Match analysis in such situations can be of immense value, allowing for game specific preparation.

Video recordings can offer coaches the chance to observe situations more closely and analyse how and why decisions were made. It provides a permanent record of performance which can be used for long-term monitoring and gives immediate feedback which can be watched repeatedly. Video can be stored for subsequent analysis and editing, recording details that might otherwise be missed during live performance, utilising freeze frame, and slow-motion capabilities that allow for detailed analysis. Video has a natural appeal as a means of communication with performers and can offer an additional source of evidence to enhance observational skills (Robertson, 1999).

Hand notation involves devising a shorthand coding system, so that events can be recorded quickly but accurately. The major emphasis on this type of system is on the gathering of descriptive data. Problems associated with hand notation, as detailed by Sanderson and Way (1977) such as processing time and accuracy, can be minimised by the additional use of video analysis. The use of video recording when using hand notation systems to evaluate performance can reduce inaccurate data input and minimise the required learning time of hand notation systems (Robertson, 1999). For these reasons, video recording combined with hand notation have been chosen as the method for analysing penalty corners in this study.

\section{Method}

The information available during a game is diverse and extensive. Continuous action and a dynamic environment make objective data collection difficult. Any quantitative analysis must be structured. Franks and Goodman (1984) outlined three steps in forming an analysis system: 
1) Describe the sport from a general level to a specific focus.

2) Prioritise key factors in performance.

3) Devise a recording method that is efficient and easy to learn.

Steps one and two can both be done by creating a flowchart or logical structure of the game itself. This means defining the possible actions in the game and linking these actions with the possible outcomes, thus describing the sequential path the game can take. Franks and Goodman (1984) describe field hockey as a two-state model. Either "our" team has possession or the opposing team has possession of the ball (Figure 1).

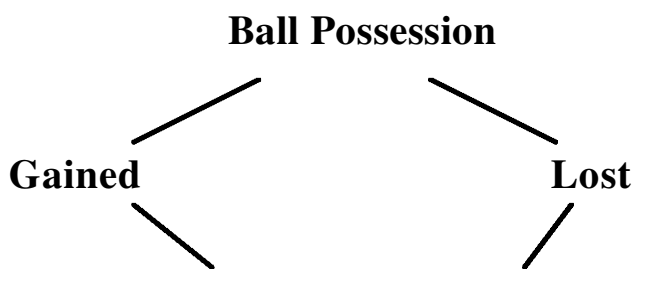

Where was it gained/lost?

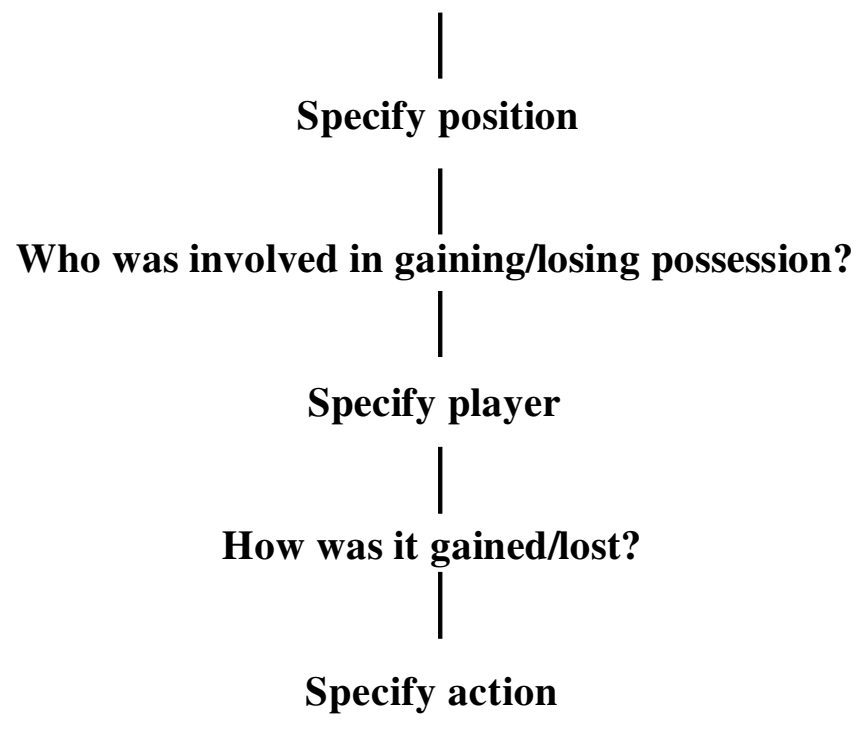

Figure 1: Hughes and Franks (1997), page 27. Hierarchical structure of a model for representing events that take place in team games, such as field hockey.

From this flowchart two tables were developed to allow a tally system to be used (Tables 1 and 2). These tables were split into successful and unsuccessful penalty corners. Success was based upon a goal being scored and the way in which it was scored. Corners were deemed unsuccessful if the ball was either put out of play or the attacking team lost possession or control of the ball.

It is from this model that the flowchart for this study has been adapted and allows for the possible actions and outcomes of penalty corners to be evaluated (Figure 2). For the purposes of this study, video footage of 250 penalty corners from the 1998 field hockey World Cup in Holland were analysed over the course of 84 games, and incidence of 2.97 per game. The video was analysed and notated using a video editing suite and hand 
notation tally system (detailed in Tables 1 and 2). The video was then edited onto another tape, so that the relevant successful and unsuccessful penalty corners could be illustrated for coaches and players.

Flowchart of possible actions and outcomes during penalty corners.

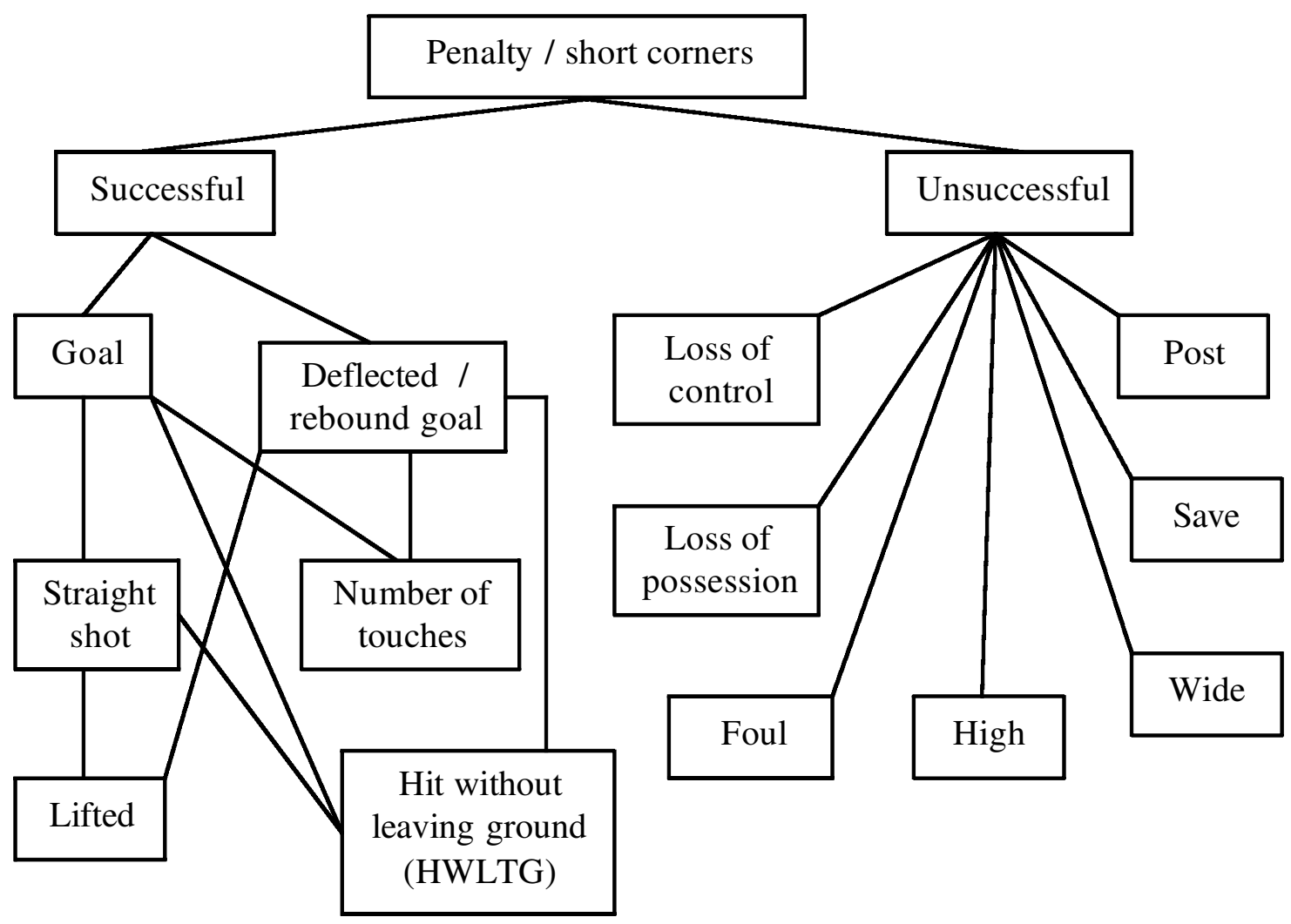

Figure 2: Flowchart of possible actions and outcomes during penalty corners, adapted from Franks and Goodman's (1984) two-state model of possession in field hockey.

Table 1: Successful penalty corners

\begin{tabular}{lccccc}
\hline & Dummy & Straight Shot & Lifted & HWLTG & $\begin{array}{l}\text { Number } \\
\text { Touches }\end{array}$ \\
\hline Goal & & & & & \\
Deflected/Rebound & & & & \\
Goal & & & & \\
\hline
\end{tabular}


Table 2: Unsuccessful penalty corners

\begin{tabular}{|c|c|c|c|}
\hline & Unsuccessful & Shots on Target & Straight Shot \\
\hline \multicolumn{4}{|l|}{ Save } \\
\hline \multicolumn{4}{|c|}{ Loss of Possession } \\
\hline \multicolumn{4}{|c|}{ Loss of Control } \\
\hline \multicolumn{4}{|c|}{ Foul } \\
\hline \multicolumn{4}{|l|}{ High } \\
\hline \multicolumn{4}{|l|}{ Wide } \\
\hline Post & & & \\
\hline
\end{tabular}

\section{Results}

The raw data obtained from analysing 250 penalty corners using the hand notation system are detailed in tables 3 and 4 . The ratio of successful to unsuccessful penalty corners was found to be 114:136.

Table 3 shows that the most successful goals were scored from straight shots that were either flicked or undercut. These types of shots are executed so quickly that the defensive players have little time to block them. Lifted shots on goal are much more difficult for the defensive opponents to gain control over and are more likely to go unchallenged into the goal mouth. Penalty corners performed using dummy shots, although a successful strategy, are not as effective as the straight shot. The results show that penalty corners where the ball is hit without leaving the ground, are more likely to result in a successful goal less often, compared to when the ball is lifted.

Balls hit without leaving the ground will usually be blocked by a defender or saved by the goalie far more often than a lifted ball.

The average number of touches which resulted in a straight shot/outright goal, was found to be three. When a greater number of touches were performed in successful penalty corners, it usually resulted in a deflected or rebound goal (Table 3).

\section{Table 3: Data of successful penalty corners.}

\begin{tabular}{llllll}
\hline & Dummy & Straight Shot & Lifted & HWLTG & $\begin{array}{l}\text { Number of } \\
\text { Touches }\end{array}$ \\
\hline Goal & 17 & 30 & 25 & 12 & 3 \\
$\begin{array}{l}\text { Deflected/Rebound } \\
\text { Goal }\end{array}$ & 3 & 5 & 11 & 11 & 6 \\
\hline
\end{tabular}

Table 4 shows that unsuccessful goals occurred most often when the ball was saved or when the attacker lost possession. This loss of possession usually occurred when the attacker lost control of the ball due to a poor stick stop or substandard pass. Initial 
viewing would suggest that most shots that were on target, resulted in a save by either the goalkeeper or a defender. These shots were usually hit without the ball leaving the ground, thus illustrating the effectiveness of lifted shots. Attempts on goal that were either played high or wide were usually due to a poorly performed lifted shot. Table 4, which shows unsuccessful penalty corners, does not define dummy shots. Dummy shots were not used very often in the execution of corners but when they were, they were always successful. Since the penalty corners analysed were extracted from hockey matches of the highest standard, the dummy shot tactic was performed with such precision that they always resulted in a goal. In lower levels of hockey, dummy shots at penalty corners are seldom practised as they are a very high risk tactic unless performed to perfection.

Table 4: Data of unsuccessful penalty corners.

\begin{tabular}{llll}
\hline & Unsuccessful & Shots on Target & Straight Shot \\
\hline Save & 28 & 9 & 5 \\
Loss of Possession & 20 & 4 & 2 \\
Loss of Control & 15 & 0 & 2 \\
Foul & 5 & 1 & 0 \\
High & 14 & 0 & 4 \\
Wide & 15 & 0 & 3 \\
Post & 4 & 3 & 2 \\
\hline
\end{tabular}

\section{Discussion and conclusions}

The pie charts in Figure 3 illustrate the data from successful penalty corners in an athlete friendly format. It highlights the effectiveness of straight shot, lifted balls and the use of dummy shots.

This information could be used by the coach and players to concentrate a portion of training time to perfecting these skills. These skills should be developed in players involved in taking penalty corners so that every corner could be a potential goal scoring opportunity. Coaches should encourage manoeuvres which are likely to lead to goal scoring and discourage those manoeuvres which are not. This may involve helping players to improve skills involved in short corners and eliminate unsuccessful skills which such analysis has proved are ineffective for goalscoring.

This analysis was based upon field hockey played at international level. These recommendations therefore, are best suited to players and teams of a comparable level. High level field hockey tends to be played at a very fast pace, where tactical decisions and strategies to deceive the opposition need to be performed instinctively and with confidence. At lower levels of field hockey, adjustments would need to be made to the advice given to allow for the lower skill levels.

During the tournament analysed penalty corners occurred only 2.97 times on average a game, or 1.48 times per team, per game. This resulted in 1.35 average goals scored a game, giving a success rate of 0.68 goals scored on average per team per game. The penalty corner can therefore provide a valuable goal scoring device. The ability to perform penalty corners well can provide the determining factor in winning a match, so increased ability in performance is vital for the successful team. 


\section{Successful Deflected/ Rebound Goals}

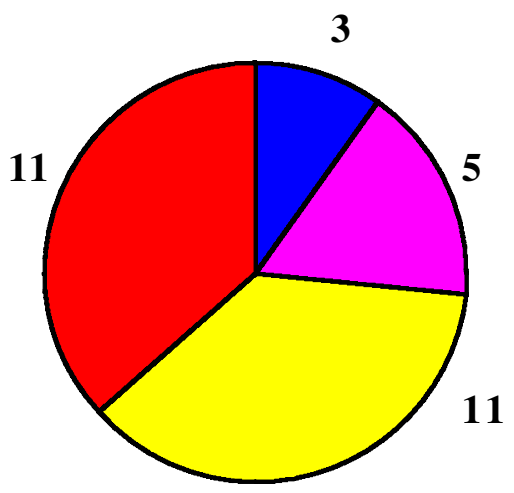

Average number of touches $=6$

\section{Successful}

Goals

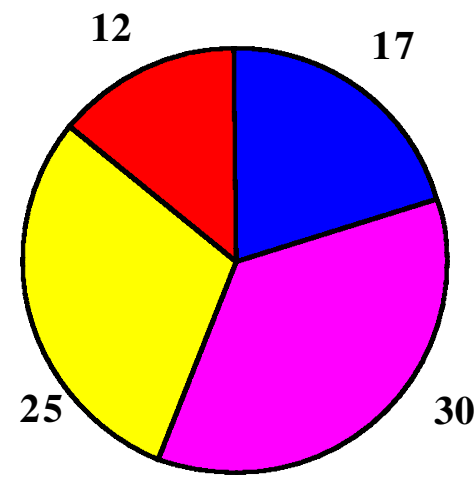

Average number of touches $=3$

Dummy

DStraight Shot

$\square$ Lifted

口 HWLTG

Fig 3: Pie charts illustrating the data obtained from successful penalty corners.

\section{References}

Franks I M and Goodman D (1984). A hierarchical approach to performance analysis. SPORTS, June.

Franks I M, Goodman D and Miller G (1983). Analysis of performance: qualitative or quantitative. SPORTS, March.

Hastie P A (1990). Models of videotape use in sports settings. Physical Education Review, 13 (2), 101-108.

Hughes, M.D., and Franks, I.M. (1997). Notational Analysis of Sport. London: E. \& F. N. Spon.

Jadgay S (1998). Penalty Corner Conversions, Field Hockey World Cup 1998. http://www.fieldhockey.org/HockeyNet/Articles/Coaching, p23.

MacHeath J A (1987). The value of match analysis techniques to the coach. Hockey Field, November, 77-78.

Robertson K (1999). Observation, Analysis and Video. Leeds: The National Coaching Foundation. 
Sanderson F H and Way K I M (1977). The development of an objective method of game analysis in squash rackets. British Journal of Sports Medicine, 11, 188.

Templin D P and Vernacchia R A (1995). The effect of highlight music videotapes upon game performance of intercollegiate basketball players. The Sport Psychologist, 9, 41. 